Check for updates

Cite this: Chem. Commun., 2019, 55, 1607

Received 6th September 2018, Accepted 21st November 2018

DOI: $10.1039 / c 8 c c 07185 c$

rsc.li/chemcomm

\section{Generation of gold nanoclusters encapsulated in an MCM-22 zeolite for the aerobic oxidation of cyclohexane $\dagger$}

\author{
Lichen Liu, ${ }^{a}$ Raul Arenal, (D) ${ }^{\text {bcd }}$ Debora M. Meira ${ }^{e}$ and Avelino Corma (D) *a
}

\begin{abstract}
In this work, we will report the generation of Au clusters in a purely siliceous MCM-22 zeolite. The catalytic properties of these Au clusters have been tested for the selective oxidation of cyclohexane to cyclohexanol and cyclohexanone (KA-oil). The Au clusters encapsulated in the MCM-22 zeolite are highly active and selective for the oxidation of cyclohexane to KA-oil, which is superior to $\mathrm{Au}$ nanoparticles on the same support. These results suggest that $\mathrm{Au}$ clusters are highly active for the activation of oxygen to produce radical species.
\end{abstract}

Gold catalysts have been intensively studied in recent years and they have been shown to exhibit unique and remarkable catalytic performances in many reactions, including selective hydrogenation, selective oxidation, organic transformations, electrocatalysis and photocatalysis. ${ }^{1-4}$ As is well known, the catalytic behavior of Au catalysts is strongly related to their particle size. Small Au particles $(<2 \mathrm{~nm})$ have been shown to be much more active than larger Au particles in many reactions. ${ }^{5,6}$ Nevertheless, when the size of Au decreases to a subnanometric regime, the catalytic properties of Au clusters are quite distinct to Au nanoparticles due to the size-dependent electronic structures of the $\mathrm{Au}$ species. ${ }^{7}$ In our recent works, it has been demonstrated that, in some reactions, only Au clusters with a few atoms are active while neither single $\mathrm{Au}$ atoms nor $\mathrm{Au}$ nanoparticles are active. ${ }^{8,9}$ However, due to their low stability and strong tendency for agglomeration, naked subnanometric $\mathrm{Au}$ clusters with open surface sites have been barely reported. ${ }^{10,11}$

\footnotetext{
${ }^{a}$ Instituto de Tecnología Química, Universitat Politècnica de València-Consejo Superior de Investigaciones Cientificas (UPV-CSIC), Av. de los Naranjos $s / n$, 46022 Valencia, Spain.E-mail: acorma@itq.upv.es

${ }^{b}$ Laboratorio de Microscopias Avanzadas, Instituto de Nanociencia de Aragon, Universidad de Zaragoza, Mariano Esquillor Edificio I + D, 50018 Zaragoza, Spain ${ }^{c}$ ARAID Foundation, 50018 Zaragoza, Spain

${ }^{d}$ Instituto de Ciencias de Materiales de Aragon, CSIC-Universidad de Zaragoza, C/Pedro Cerbuna 12, 50009 Zaragoza, Spain

${ }^{e}$ European Synchroton Radiation Facility, 6 Rue Jules Horowitz, Grenoble, BP 156, F-38042, France

$\dagger$ Electronic supplementary information (ESI) available. See DOI: 10.1039/c8cc07185c
}

Using organometallic Au complexes as precursors, Au clusters can be generated in zeolites. ${ }^{12}$ This strategy, however, is restricted for processes with activation and reaction conditions that do not require higher temperature.

Considering the low thermal stability of $\mathrm{Au}$ particles, the encapsulation of Au species in zeolites should be a promising approach to enhance their stability. ${ }^{13}$ The well-defined pore structures of zeolites can provide effective protection for $\mathrm{Au}$ species under reaction conditions and introduce, in some cases, size-selective catalytic properties. During the past several years, the encapsulation of $\mathrm{Au}$ into zeolite crystallites has been achieved by several methods. Au nanoparticles (2-3 nm) can be encapsulated in silicalite-1 with intraparticle voids and mesopores through a simple impregnation method. ${ }^{14}$ Recently, Iglesia and his co-workers reported a method to generate small $\mathrm{Au}$ nanoparticles (1-2 nm) in Al-containing zeolites with high thermal stability and resistance to poison molecules in catalytic reactions. ${ }^{15}$ In our recent work, subnanometric Pt single atoms and Pt clusters were encapsulated in MCM-22 through a $2 \mathrm{D}$ to 3D transformation process. These subnanometric Pt species show excellent stability even at a high temperature (as high as $\left.550{ }^{\circ} \mathrm{C}\right) .{ }^{16,17}$

In this work, by the transformation of a two-dimensional zeolite into a three-dimensional zeolite, naked subnanometric $\mathrm{Au}$ clusters can also be generated in purely siliceous MCM-22, and the resultant material presents an excellent catalytic activity and selectivity for the selective oxidation of cyclohexane with $\mathrm{O}_{2}$ to produce cyclohexanol and cyclohexanone (KA-oil).

As shown in Fig. 1, the Au@MCM-22 sample was prepared through a similar procedure to that we had reported in our recent work for the preparation of Pt@MCM-22. Au clusters dispersed in DMF are prepared firstly as the metal precursor. Subsequently, the Au clusters are incorporated between the MWW layers in ITQ-1 during a swelling process. After the removal of the organics in the swollen material, Au species are encapsulated by the final MCM-22 zeolite, leading to the formation of Au@MCM-22-S. However, the Au loading in the Au@MCM-22-S sample was only $c a$. $0.025 \mathrm{wt} \%$. Then, in order to improve the 


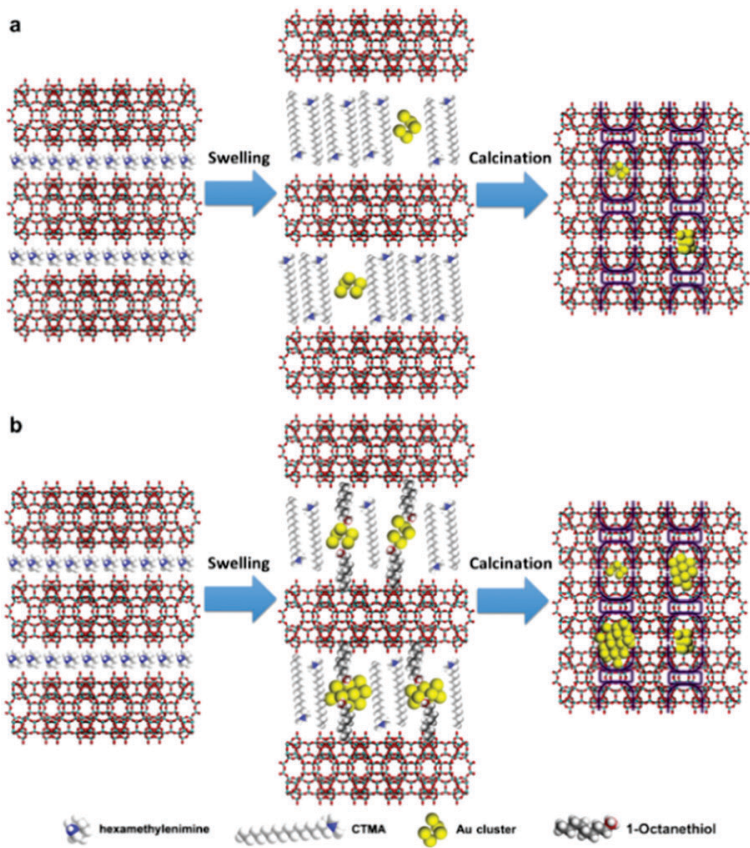

Fig. 1 Schematic illustration of the incorporation of Au nanoclusters into a zeolite through the transformation of a 2D zeolite into a 3D structure (MCM-22). (a) The incorporation of Au clusters in MCM-22 by swelling of the ITQ-1 with a surfactant and a DMF solution containing Au clusters. (b) The incorporation of Au clusters in MCM-22 by swelling of the ITQ-1 with a surfactant, 1-octanethiol and a DMF solution containing Au clusters.

$\mathrm{Au}$ loading in the final Au@MCM-22 material, 1-octanethiol is added here to the swelling mixture (see Fig. 1b), since the strong $\mathrm{Au}-\mathrm{S}$ bonding interaction may help the incorporation of Au species into the MWW layers. ${ }^{18,19}$ As a result, an Au@MCM22-L sample was obtained with an Au loading of $c a .0 .11 \mathrm{wt} \%$. In principle, the introduction of 1-octanethiol can also be applied to the incorporation of other metals into MCM-22 by our strategy, leading to a higher metal loading and maintenance of good metal dispersion in the zeolite at the same time.

Firstly, the Au species in the Au@MCM-22-S and Au@MCM22-L samples have been characterized by electron microscopy. In the low-magnification STEM image of Au@MCM-22-S (see Fig. 2a), Au nanoclusters with particle size ranging from $0.5 \mathrm{~nm}$ to $1 \mathrm{~nm}$ can be seen together with a few Au nanoparticles of 1-2 nm. The atomic structure of the subnanometric Au clusters was revealed by the high-resolution STEM images (see Fig. 2b and c). As it can be seen, Au nanoclusters with less than 15 atoms are located in the supercages or surface "cups" of MCM-22. More high-resolution STEM images of various subnanometric Au species are shown in Fig. S1 (ESI $\dagger$ ), including single $\mathrm{Au}$ atom and $\mathrm{Au}$ clusters with less than 10 atoms. Considering the size of the supercages in MCM-22, the maximum size of the Au clusters is below $1 \mathrm{~nm}$, corresponding to the $\mathrm{Au}$ clusters with less than 15 atoms. In the case of Au@MCM-22-L, Au nanoclusters around $1 \mathrm{~nm}$ can also be observed in the STEM images (see Fig. 2d-f). The particle size of the $\mathrm{Au}$ species in the Au@MCM-22-L sample is slightly larger than that in the Au@MCM-22-S sample.
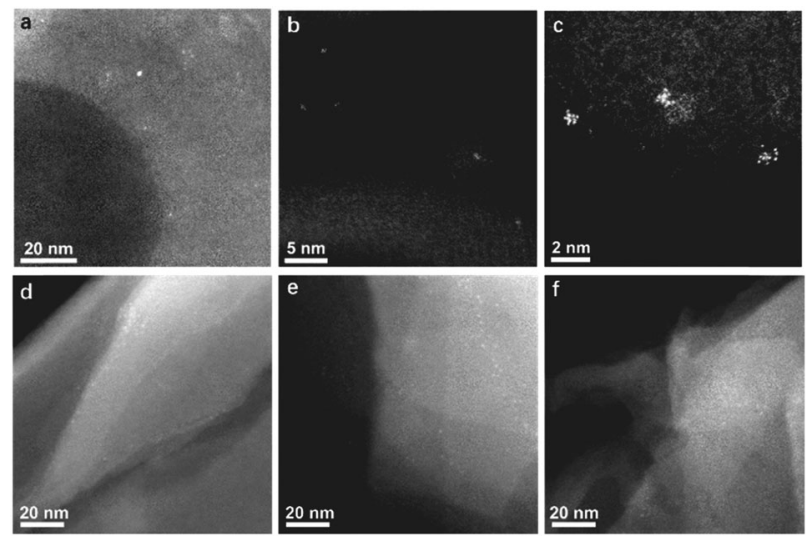

Fig. 2 Electron microscopy characterization on the Au@MCM-22 samples. (a) STEM image of the AuaMCM-22-S sample. (b and c) High-resolution STEM images of the subnanometric Au clusters observed in the AuaMCM22-S sample. (d-f) STEM images of the Au@MCM-22-L sample, showing the presence of both subnanometric $\mathrm{Au}$ clusters and $\mathrm{Au}$ nanoclusters around $1 \mathrm{~nm}$.

To gain more information on the structures of the $\mathrm{Au}$ clusters in the Au@MCM-22 samples, various spectroscopic characterization tools were used to study them. The coordination environment and average size of the Au species in the Au@MCM-22 samples have been studied by X-ray absorption spectroscopy (XAS). The chemical states of the Au species in the Au@MCM-22-S and Au@MCM-22-L samples are studied by $\mathrm{X}$-ray absorption near edge structure (XANES) analysis. As displayed in Fig. 3a, both Au@MCM-22-S and Au@MCM-22-L show similar XANES spectrum to the reference metallic $\mathrm{Au}$, indicating that the Au nanoclusters exist in the metallic state in the MCM-22 zeolite. Furthermore, the coordination environment of the Au species has also been studied by extended X-ray absorption fine structure (EXAFS) analysis. As can be seen in Fig. $3 \mathrm{~b}$, the fitting results of the EXAFS spectra are shown in Table 1. The Au-Au coordination number in Au@MCM-22-S and $\mathrm{Au} @ \mathrm{MCM}-22-\mathrm{L}$ is 7.0 and 7.7, respectively. Thus, the average size of the Au species in Au@MCM-22-S is $\sim 1 \mathrm{~nm}$ and $\sim 1.2 \mathrm{~nm}$ in Au@MCM-22-L, which is consistent with the electron microscopy images in Fig. 2, if one takes into account the impact of the larger particles on the average $\mathrm{Au}-\mathrm{Au}$ coordination number
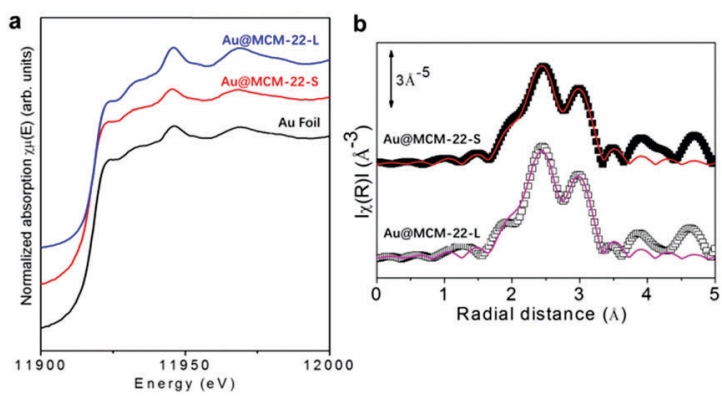

Fig. 3 (a) XANES spectra of the Au@MCM-22-L and Au@MCM-22-S samples and the Au foil reference. (b) Fourier transform of $k^{2}$-weighted EXAFS spectra and the fitting curves for the Au@MCM-22-L and Au@MCM22-S samples. 
Table 1 Fitting results of the EXAFS spectra for the Au@MCM-22-L and Au@MCM-22-S samples. $S_{0}^{2}=0.8$ and $E_{0}=5 \mathrm{eV}$. More fitting details are shown in the ESI

\begin{tabular}{llllll}
\hline Sample & $\mathrm{CN}_{\mathrm{Au}-\mathrm{Au}}$ & $\sigma^{2}\left(\AA^{2}\right)$ & $R(\AA)$ & $\mathrm{CN}_{\text {ref }}$ & $R_{\text {ref }}(\AA)$ \\
\hline Au@MCM-22-L & $7.7 \pm 0.6$ & $0.008 \pm 0.001$ & $2.853 \pm 0.003$ & 12 & 2.88470 \\
Au@MCM-22-S & $7.0 \pm 0.6$ & & $2.855 \pm 0.004$ & &
\end{tabular}

obtained from the EXAFS fitting results. ${ }^{20,21}$ Then, by combining the results of electron microscopy and XAS, it can be deduced that, both Au@MCM-22-L and Au@MCM-22-S consist of a mixture of subnanometric $\mathrm{Au}$ clusters and small $\mathrm{Au}$ nanoparticles (1-2 nm). Furthermore, the percentage of subnanometric Au clusters in the Au@MCM-22-S sample is higher than that in the Au@MCM-22-L sample according to the EXAFS fitting results.

For comparison, a sample consisting of $\mathrm{Au}$ nanoparticles supported on MCM-22 (containing $0.1 \mathrm{wt} \%$ of Au, denoted as AuNP/MCM-22) was also prepared by a deposition-precipitation method (see the ESI $\dagger$ for experimental details). As shown in Fig. S2 (ESI $\dagger$ ), the particle size of the Au nanoparticles in AuNP/ MCM-22 ranges from 1.2 to $2.5 \mathrm{~nm}$.

It is well known that the electronic structures of metal clusters and nanoparticles are usually associated with their optical properties. As shown in Fig. S3 (ESI $\dagger$ ), the typical surface plasmon resonance of the Au nanoparticles can be observed in the UV-vis spectrum of the AuNP/MCM-22 sample at $\sim 530 \mathrm{~nm}$. In the case of the Au@MCM-22 samples containing Au nanoclusters, we can observe the absorption bands at $\sim 615$ and $\sim 670 \mathrm{~nm}$, corresponding to the absorption bands between the HOMO and the LUMO of Au clusters with different sizes. ${ }^{22}$ At this point, based on the above structural and spectroscopic characterization results, it is confirmed that the Au nanoclusters have been generated and stabilized in MCM-22.

To test the catalytic properties of Au clusters, the oxidation of cyclohexane to cyclohexanol and cyclohexanone (KA-oil) has been carried out. It has been established in the literature that the auto-oxidation of cyclohexane is a radical-chain reaction. ${ }^{23}$ Moreover, it has also been proposed that supported Au nanoparticles are actually inert in the oxidation of cyclohexane. ${ }^{24}$ It has been demonstrated recently by our group that Au clusters are efficient catalysts for the activation of $\mathrm{O}_{2}$ and the production of radical oxygen species. ${ }^{8,9}$ Therefore, it is supposed that the Au clusters stabilized in the MCM- 22 zeolite may serve as active species for the initiation of the autocatalytic oxidation of cyclohexane to KA-oil.

In this work, the oxidation of cyclohexane was performed in a batch reactor without the addition of a radical initiator. As presented in Fig. 4a, all the samples show an induction period in the oxidation of cyclohexane, corresponding to the in situ generation of radicals under reaction conditions. The control experiments without a solid catalyst (Thermal) and the Au-free MCM-22 support show low conversion of cyclohexane after $2 \mathrm{~h}$, and the major product is cyclohexyl hydroperoxide (see Fig. 4b). Furthermore, the conversion of cyclohexane with the AuNP/MCM-22 catalyst is also very low, indicating that $\mathrm{Au}$

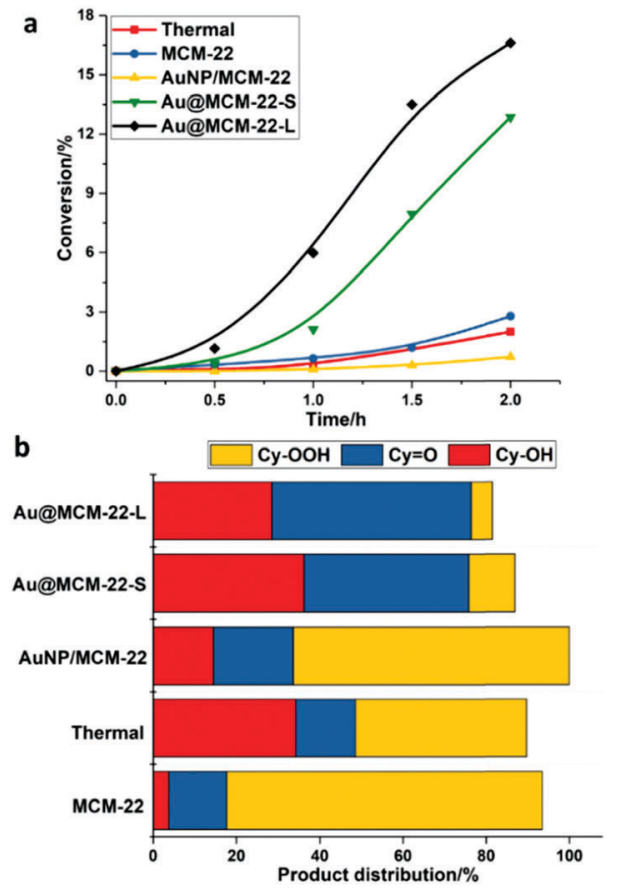

Fig. 4 Catalytic performance of the AuNP/MCM-22 and Au@MCM-22 catalysts for the aerobic oxidation of cyclohexane. (a) Time-profile of the conversion of cyclohexane with different catalysts. (b) Product distributions obtained with different catalysts after $2 \mathrm{~h}$ of reaction. Reaction conditions: $2 \mathrm{~mL}$ cyclohexane, $25 \mathrm{mg}$ solid catalyst, $150{ }^{\circ} \mathrm{C}$, and 10 bar of $\mathrm{O}_{2}$. The "thermal" test was carried out under the same conditions in the absence of a catalyst.

nanoparticles are not active for this process, which is consistent with the previous works. ${ }^{24}$

Remarkably, the kinetic curves shown in Fig. 4a show that both Au@MCM-22-S and Au@MCM-22-L containing subnanometric Au clusters show a shorter induction period $(\sim 0.5 \mathrm{~h})$ in the oxidation of cyclohexane and a much higher conversion than the previous experiments, indicating the higher activity for the generation of radicals and for the decomposition of the hydroperoxide intermediates. As we can see in Fig. $4 \mathrm{a}$, a high conversion of cyclohexane $(>12 \%)$ can be achieved with Au@MCM-22-S and Au@MCM-22-L after 2 hours and the selectivity to the desired products (cyclohexanol, cyclohexanone and cyclohexyl hydroperoxide) on both Au@MCM-22 samples is similar. Au@MCM-22-S shows a slightly higher selectivity than Au@MCM-22-L (see Fig. 4b). The evolution of the product distribution with the reaction time is similar on both $\mathrm{Au} @ \mathrm{MCM}-22$ catalysts (see Fig. S4 and S5, ESI $\dagger$ ). The yields of various products at different cyclohexane conversions are shown in Fig. S6 (ESI $\dagger$ ). It should be noted that, when the same amount of the solid catalyst (25 mg) was used, Au@MCM-22-L shows higher yields of products at the same reaction time. However, if the amount of Au species is kept the same for the two catalysts, then Au@MCM-22-S shows better performance for the oxidation of cyclohexane to KA-oil (see Fig. S7 and S8, ESI $\dagger$ ). Considering the higher percentage of subnanometric Au clusters in the Au@MCM-22-S sample, these results imply that the Au clusters with low atomicity are probably the active species instead of the ones larger than $1 \mathrm{~nm}$. 
The oxidation of cyclohexane has been proven to be initiated by the formation of cyclohexyl hydroperoxide radicals and subsequently the homolytic cleavage of cyclohexyl hydroperoxide to form free radicals. Therefore, the role of a catalyst in this reaction can be related to the acceleration of the generation of hydroperoxide radicals. ${ }^{25}$ The role of a radical intermediate in initiating the aerobic oxidation of cyclohexane has been confirmed by catalytic tests in the presence of an initiator (see Fig. S9 and S10, ESI $\dagger$ ). ${ }^{26}$ It has been observed in different systems that the activation mechanism of $\mathrm{O}_{2}$ on metal particles is related to the particle size. In the case of $\mathrm{Au}$ nanoparticles, $\mathrm{O}_{2}$ is activated into atomic oxygen species at $T>80{ }^{\circ} \mathrm{C}$. While in the case of Au clusters, $\mathrm{O}_{2}$ can be activated and transformed into radical-type species on Au clusters. ${ }^{9,27}$ The different catalytic behavior of $\mathrm{Au}$ nanoclusters and nanoparticles for the aerobic oxidation of cyclohexane is then probably related to the size-dependent $\mathrm{O}_{2}$ activation mechanism on the Au species.

We also study the stability of Au clusters for the oxidation of KA-oil. As shown in Fig. S5 (ESI $\dagger$ ), the catalyst shows good recyclability during three consecutive tests. The conversion and yields of different products are similar for the three tests. As presented in Fig. S11 (ESI $\dagger$ ), the presence of Au nanoclusters in the used catalyst is confirmed using the electron microscopy images and the agglomeration of the $\mathrm{Au}$ species into the $\mathrm{Au}$ nanoparticles is not observed, suggesting the good stability of the Au nanoclusters encapsulated in MCM-22.

In summary, we have reported the synthesis of Au@MCM-22 materials containing subnanometric Au clusters in an MCM-22 zeolite by incorporating Au species during the transformation of a $2 \mathrm{D}$ zeolite into a 3D structure. These highly stable $\mathrm{Au}$ clusters can serve as active species for the aerobic oxidation of cyclohexane into cyclohexanol and cyclohexanone without the presence of a radical initiator.

This work has been supported by the European Union through the European Research Council (grant ERC-AdG-2014-671093, SynCatMatch) and the Spanish government through the "Severo Ochoa Program" (SEV-2016-0683). The authors also thank the Microscopy Service of UPV for kind help with TEM and STEM measurements. Mr J. A. Gaona is greatly acknowledged for his very helpful assistance on the catalytic studies. The XAS data were acquired at European Synchrotron Radiation Facility. The HAADFHRSTEM studies were conducted in the Laboratorio de Microscopias Avanzadas (LMA) at the Instituto de Nanociencia de Aragon (INA)-Universidad de Zaragoza (Spain), Spanish ICTS National facility. R. A. gratefully acknowledges the support from the Spanish Ministry of Economy and Competitiveness (MINECO) through project grant MAT2016-79776-P (AEI/FEDER, UE).

\section{Conflicts of interest}

There are no conflicts to declare.

\section{Notes and references}

1 P. Claus, Appl. Catal., A, 2005, 291, 222-229.

2 T. Takei, T. Akita, I. Nakamura, T. Fujitani, M. Okumura, K. Okazaki, J. Huang, T. Ishida and M. Haruta, Adv. Catal., 2012, $55,1-126$.

3 A. S. Hashmi and G. J. Hutchings, Angew. Chem., Int. Ed., 2006, 45, 7896-7936.

4 L. Liu and A. Corma, Chem. Rev., 2018, 118, 4981-5079.

5 M. Valden, X. Lai and D. W. Goodman, Science, 1998, 281, $1647-1650$

6 B. Hvolbæk, T. V. W. Janssens, B. S. Clausen, H. Falsig, C. H. Christensen and J. K. Nørskov, Nano Today, 2007, 2, 14-18.

7 J. Oliver-Meseguer, J. R. Cabrero-Antonino, I. Dominguez, A. LeyvaPerez and A. Corma, Science, 2012, 338, 1452-1455.

8 A. Corma, P. Concepcion, M. Boronat, M. J. Sabater, J. Navas, M. J. Yacaman, E. Larios, A. Posadas, M. A. Lopez-Quintela, D. Buceta, E. Mendoza, G. Guilera and A. Mayoral, Nat. Chem., 2013, 5, 775-781.

9 M. Boronat, A. Leyva-Perez and A. Corma, Acc. Chem. Res., 2014, 47, 834-844.

10 S. Yamazoe, K. Koyasu and T. Tsukuda, Acc. Chem. Res., 2014, 47, 816-824.

11 R. Jin, C. Zeng, M. Zhou and Y. Chen, Chem. Rev., 2016, 116, 10346-10413.

12 J. C. Fierro-Gonzalez, Y. Hao and B. C. Gates, J. Phys. Chem. C, 2007, 111, 6645-6651.

13 M. T. Bore, H. N. Pham, E. E. Switzer, T. L. Ward, A. Fukuoka and A. K. Datye, J. Phys. Chem. B, 2005, 109, 2873-2880.

14 J. Mielby, J. O. Abildstrom, F. Wang, T. Kasama, C. Weidenthaler and S. Kegnaes, Angew. Chem., Int. Ed., 2014, 53, 12513-12516.

15 T. Otto, S. I. Zones and E. Iglesia, J. Catal., 2016, 339, 195-208.

16 L. Liu, U. Diaz, R. Arenal, G. Agostini, P. Concepcion and A. Corma, Nat. Mater., 2017, 16, 132-138.

17 L. Liu, D. N. Zakharov, R. Arenal, P. Concepcion, E. A. Stach and A. Corma, Nat. Commun., 2018, 9, 574.

18 Y. Xue, X. Li, H. Li and W. Zhang, Nat. Commun., 2014, 5, 4348.

19 E. Pensa, E. Cortes, G. Corthey, P. Carro, C. Vericat, M. H. Fonticelli, G. Benitez, A. A. Rubert and R. C. Salvarezza, Acc. Chem. Res., 2012, 45, 1183-1192.

20 A. Shivhare, D. M. Chevrier, R. W. Purves and R. W. J. Scott, J. Phys. Chem. C, 2013, 117, 20007-20016.

21 J. T. Miller, A. J. Kropf, Y. Zha, J. R. Regalbuto, L. Delannoy, C. Louis, E. Bus and J. A. van Bokhoven, J. Catal., 2006, 240, 222-234.

22 M. Zhu, C. M. Aikens, F. J. Hollander, G. C. Schatz and R. Jin, J. Am. Chem. Soc., 2008, 130, 5883-5885.

23 I. Hermans, Liquid Phase Aerobic Oxidation Catalysis-Industrial Applications and Academic Perspectives, ed. S. Stahl and P. Alsters, 2015.

24 B. P. C. Hereijgers and B. M. Weckhuysen, J. Catal., 2010, 270, $16-25$.

25 I. Hermans, P. A. Jacobs and J. Peeters, Chem. - Eur. J., 2006, 12, 4229-4240.

26 M. Conte, X. Liu, D. M. Murphy, K. Whistonb and G. J. Hutchings, Phys. Chem. Chem. Phys., 2012, 14, 16279-16285.

27 L. Qian, Z. Wang, E. V. Beletskiy, J. Liu, H. J. Dos Santos, T. Li, M. D. Rangel, M. C. Kung and H. H. Kung, Nat. Commun., 2017, 8,14881 . 